\title{
Multivariable Saturation Compensator Designs through Singular Value Decompositions
}

\author{
K. Hui and C.W. Chan \\ Department of Mechanical Engineering, University of Hong Kong, Pokfulam Road, Hong Kong \\ Email: khui@hkumea.hku.hk

\section{URL: http://hkumea.hku.hk/ khui}

\section{Abstract}

Using a general saturation compensation framework for multi-input multi-output control systems subject to actuator constraints, a simple expression relating the singular values of the uncompensated system, the compensator and the compensated system is established. This singular value decomposition offers numerous possibilities in shaping the desired responses for the saturation compensated system. Two specific practical designs are proposed from the SVD analysis. Another two possibilities are exposed for further theoretical development. Simulated example demonstrates the feasibility and practicality of the proposed designs.

\section{Introduction}

In spite of the propositions of some unified compensation frameworks in the literature for multi-input multi-output (MIMO) control systems subject to actuator saturations [5, 9], hitherto only a few specific designs of the compensators are proposed $[1,7,8]$. Most of these designs are based on time-domain considerations or optimization methods. The application of MIMO frequency domain techniques to constrained MIMO systems is rarely reported.

Instead of the skeletal framework by [5], a special formulation of the compensators [3] is adopted below in order to establish a fundamental relationship between the uncompensated system, the compensator, and the compensated system. This shall enable analyses of the compensated system, as well as designs of compensators, in the settings of MIMO frequency domain properties. By appropriate shaping of the singular values, issues of satisfactory performance, robustness and stability of the compensated systems, can all be tackled simultaneously.

In $\$ 2$ below, a special formulation of general MIMO compensator is adopted to establish the fundamental relationship between the various sensitivity functions. Singular value analyses of the sensitivity functions are performed in $\$ 3$, together with two specific practical compensator designs. Another two theoretical expositions are presented in $\$ 4$. A simulated example illustrates the achievable results using the practical designs in $\$ 5$.

\section{Compensation Framework}

Denote $R^{m \times n}$ the set of all real matrices with $m$-rows by $n$ columns. Then a linear MIMO plant can be described by

$$
\left.G: \quad \begin{array}{l}
\dot{x}(t)=A x(t)+B u(t) \\
y(t)=C x(t)+D u(t)
\end{array}\right\}
$$

where $x \in R^{m \times 1}$ is the system state, $y \in R^{m \times 1}$ the plant output, $u \in R^{m \times 1}$ the plant input. $A \in R^{m \times m}, B \in R^{m \times n}, C \in R^{m \times m}, D \in R^{n \times n}$. As usual, assume $(A, B)$ controllable and $(A, C)$ observable. The transfer function of the plant, in Laplace transform variable $s$, is $G(s)=C[s I-A]^{-1} B+D$.

The linear controller is described by

$$
\left.K: \begin{array}{l}
\dot{x}_{c}(t)=A_{c} x_{c}(t)+B_{c} e(t) \\
v(t)=C_{c} x_{c}(t)+D_{c} e(t)
\end{array}\right\}
$$

where $x_{c} \in R^{k \times 1}$ is the controller state, $v \in R^{n \times 1}$ the controller output. $A_{c} \in R^{k \times k}, B_{c} \in R^{k \times n}, C_{c} \in R^{n \times k}, D_{c} \in R^{n \times n}$. The control

error is $\quad e(s)=F_{w}(s) w(s)-y(s)$

where $e \in R^{n \times 1}, w \in R^{n \times 1}$ is the reference setpoint. $F_{w}(s)$ is the transfer function of the setpoint filter, so that (2.2)-(2.3) forms a 2-degrees of freedom controller, whose transfer function is $K(s)=C_{c}\left[s I-A_{c}\right]^{-1} B_{c}+D_{c}$.

The $i$-th actuator output is constrained by

$$
u_{i}(t)=\operatorname{sat}\left[v_{i}\right]=\left\{\begin{array}{lr}
u_{i \max }, & v_{i} \geq u_{i \max } \\
v_{i}, & u_{i \min }<v_{i}<u_{i \max } \\
u_{i \min }, & v_{i} \leq u_{i \min }
\end{array}\right.
$$

where limits $\left\{u_{i \max }, u_{i \min }\right\}$ are assumed known, $\forall i=1 \cdots n$.

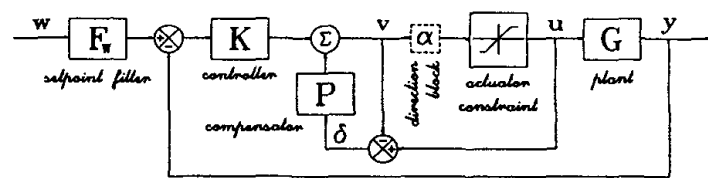

Fig.1 MIMO systems with saturation compensator The saturation compensated system is shown in Fig.1, which is a special form of a unified framework [5]. The controller output in Fig. 1 is given by

$$
\begin{array}{cc} 
& v=K\left[F_{w} w-y\right]+P \delta \\
\text { where } & \delta(t) \triangleq u(t)-v(t)
\end{array}
$$

and the compensator is defined as

$$
\left.P: \quad \begin{array}{l}
\dot{x}_{P}(t)=A_{P} x_{P}(t)+B_{P} \delta(t) \\
v_{P}(t)=C_{P} x_{P}(t)+D_{P} \delta(t)
\end{array}\right\}
$$

with $v_{P}\left(\in R^{n \times 1}\right)=P \delta$, being the compensator output. $A_{P} \in R^{p \times p}$, $B_{P} \in R^{p \times n}, C_{P} \in R^{n \times p}, D_{P} \in R^{n \times n}$. Again let $\left(A_{P}, B_{P}\right)$ controllable and $\left(A_{P}, C_{P}\right)$ observable, so that there is no hidden dynamics within $P$. Except integrators, all eigenvalues of $A_{P}$ are assumed asymptotically stable. The transfer function

$$
P(s)=C_{P}\left[s I-A_{P}\right]^{-1} B_{P}+D_{P}
$$

needs not be diagonal. In which case, there will be coupled compensation from cross-saturation terms. 


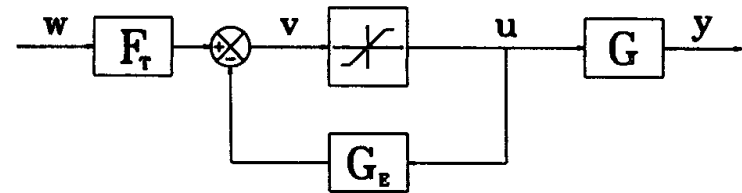

Fig.2 Equivalent system of Fig. 1 for analysis and design Using (2.6) and from $y=G u,(2.5)$ becomes

$v=K F_{w} w-K G u+P[u-v]=F_{T} w-G_{E} u$

where $\left.\begin{array}{l}F_{T}(s)=[I+P(s)]^{-1} K(s) F_{w}(s) \\ G_{E}(s)=[I+P(s)]^{-1}[K(s) G(s)-P(s)]\end{array}\right\}$

The closed-loop system representing (2.9) is shown in Fig.2. Obviously $[I+P(s)]$ must be invertible for $(2.10)$ to exist. Its system output is given by

$$
\left.\begin{array}{c}
y \Delta y_{0}+\Delta y ; \quad y_{0}=[I+G K]^{-1} G K F_{w} w \\
\Delta y=[I+G K]^{-1} G[I+P] \delta
\end{array}\right\}
$$

$y_{0}$ is the unconstrained linear system response and $\Delta y$ is the output variation due to the saturation disturbance $\delta$. Even if the system is uncompensated, i.e., $P=0, \Delta y$ in (2.11) is still nonzero unless $\delta \equiv 0$. Subsequently, the purpose of saturation compensation is to alleviate, or minimize, the variations of $\Delta y$ by proper selections of $P$, assuming that the linear system design $[I+G K]^{-1} G K F_{w^{\prime}}$ is satisfactory. For asymptotically stable saturation systems, $\delta(t) \rightarrow 0$ as $t \rightarrow \infty$.

Since (2.7) is always realizable, the only conditions so far $P$ has to satisfy are

(P1) poles of $[I+G K]^{-1} G[I+P]$ are asymptotically stable;

(P2) poles of $[I+P]^{-1}$ are asymptotically stable.

(P1) is to ensure $\Delta y(t) \rightarrow 0$ as $t \rightarrow \infty$, so that the saturation effect dies out and $y(t) \rightarrow y_{0}(t)$ as $t \rightarrow \infty$. $y_{0}(t)$ may not approach $w(t)$ in the linear system design. (P2) is only necessary when global stability of $G_{E}$ in $(2.10)$ is being considered.

For the system configuration shown in Fig.2, it is clear that issues on performance, stability and robustness of the compensated system is entirely vested with the equivalent system $G_{E}$. Results from analyses of $G_{E}$ shall provide valuable information on how to design the compensator $P$. From (2.10),

$$
\left[I+G_{E}(s)\right]=[I+P(s)]^{-1}[I+K(s) G(s)]
$$

Define the sensitivity functions

$S_{K G} \Delta[I+K G]^{-1}, S_{G E} \Delta\left[I+G_{E}\right]^{-1}, S_{P} \Delta[I+P]^{-1}$

Thus (2.12) gives

$$
S_{G E}=S_{K G} S_{P}^{-1}
$$

Notice that $S_{K G}$ is the input sensitivity of the linear system [6], in contrast to the output sensitivity, $S_{G K}=[I+G K]^{-1}$. Unless the system is normal $(G K=K G)$, otherwise $S_{K G} \neq S_{G K}$. Furthermore, linear system designs mostly consider $S_{G K}$; which leaves behind some space for maneuvering when $S_{K G}$ differs significantly from $S_{G K}$.

(2.14) relates the sensitivity function of the compensated system with that of the uncompensated one, and that of the compensator. This fundamental relationship shall be repeatedly cracked to yield results for the design of saturation compensators in the following sections.

\section{Analysis of Singular Values}

This section looks into the sensitivity functions, established above in (2.14), and their singular value decompositions. Discussions on characteristic loci, $\lambda\left[G_{E}(j \omega)\right]$, of the compensated system using (2.12), are omitted in this work because firstly $K G(j \omega)$ shares the same nonzero eigenvalues as $G K(j \omega)$ and secondly, analysis of characteristic loci does not always give conclusive results.

A simple compensator is immediately obtained from a usual design consideration to minimize measurement noise propagation [6], that the sensitivity function should be around unity. Pushing this idea to the limit with the equivalent system, in requiring that every singular value $\sigma\left[S_{G E}\right]=1$, then from (2.14),

$$
\begin{array}{cc}
S_{G E} \equiv I: & I=S_{K G} S_{P}^{-1} \Rightarrow S_{P}=S_{K G} \\
P=K G & =K
\end{array}
$$
giving

If $K G$ is neutrally or asymptotically stable without unstable pole-zero cancellations, then $P$ in (3.2) is a perfectly legitimate choice of a compensator for the configuration shown in Fig.1. Both conditions (P1)-(P2) are automatically satisfied by the linear designs. A practical example of $P$ given by (3.2) is demonstrated in $\S 5$.

Obviously, the requirement that all singular values of $S_{G E}$ be unity is too extreme, nor is it necessary. Let $\bar{\sigma}\left[S_{K G}\right]$ ( $=\max \left\{\sigma\left[S_{K G}\right]\right\}$ ) be as shown in Fig.3, with an $H_{\infty}$-norm

$$
\left\|S_{K G}(j \omega)\right\|_{\infty} \triangleq \sup _{\omega}\left\{\bar{\sigma}\left[S_{K G}(\omega)\right]\right\}=+k_{s}
$$

at frequency $\omega_{s}$. If an ideal filter can be found so that it just removes all the gains of $\bar{\sigma}\left[S_{K G}(j \omega)\right]>0 \mathrm{~dB}$ (Fig.3), then $\bar{\sigma}\left[S_{G E}(j \omega)\right] \leq 1, \forall \omega$. This modification can be expected to produce satisfactory saturation compensation results.

To shape $\sigma\left[S_{G E}\right]$, let the SVDs be [6]:

$$
\left.\begin{array}{c}
S_{K G}=U_{K G} \Sigma_{K G} V_{K G}^{H} \quad, \quad S_{P}=U_{P} \Sigma_{P} V_{P}^{H} \\
S_{G E}=U_{G E} \Sigma_{G E} V_{G E}^{H}
\end{array}\right\}
$$

for some unitary matrices $\left\{U_{K G}, V_{K G}, U_{P}, V_{P}, U_{G E}, V_{G E}\right\}$ and singular values $\left\{\Sigma_{K G}, \Sigma_{P}, \Sigma_{G E}\right\}$. The complex conjugate transpose is denoted by $(\cdot)^{H}$. From (2.14),

$$
\begin{aligned}
S_{G E} & =\left[U_{K G} \Sigma_{K G} V_{K G}^{H}\right]\left[U_{P} \Sigma_{P} V_{P}^{H}\right]^{-1} \\
& =U_{K G} \Sigma_{K G} V_{K G}^{H} V_{P} \Sigma_{P}^{-1} U_{P}^{H}
\end{aligned}
$$

If the same compensator $p_{0}(s)$ is used for each loop without cross-compensation, then the overall compensator is

so that $\quad \Sigma_{P}=\sigma_{p} I$

where $\sigma_{P}^{-1}=\left|1+p_{0}(j \omega)\right|$. Thus, (3.5) becomes

$$
\begin{array}{cc} 
& S_{G E}=U_{K G}\left[\sigma_{P}^{-1} \Sigma_{K G}\right]\left[U_{P} V_{P}^{H} V_{K G}\right]^{H} \\
\text { giving } & \Sigma_{G E}=\sigma_{P}^{-1} \Sigma_{K G}
\end{array}
$$


In this manner, each $\sigma\left[S_{K G}\right]$ is modified by the same gain reduction $\sigma_{P}$. The restriction that each $\sigma\left[S_{G E}\right]$ cannot be separately specified shall be discussed in $\S 4$.

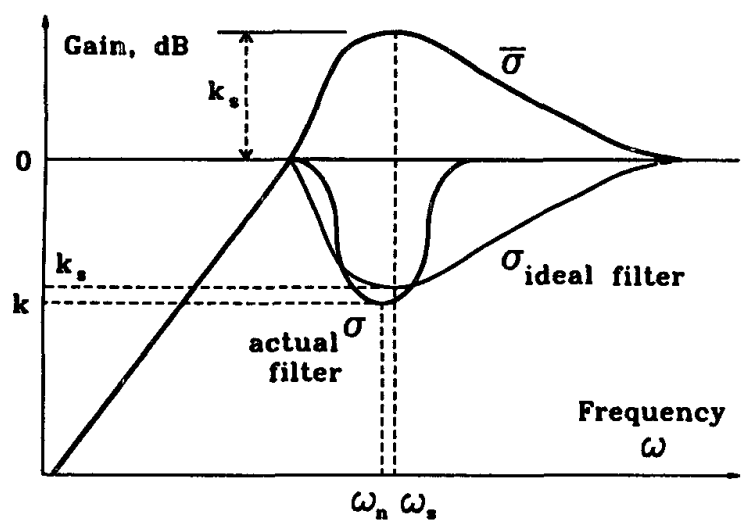

Fig.3 Determination of notch filter parameters

More frequently, prefect cancellations as depicted by the ideal filter (Fig.3) is not realistic and some compromise has to be settled for. One approximation to the ideal shape would be a notch filter $F_{n}^{-1}(s)$, defined as

$$
F_{n}^{-1}(s)=\frac{\left(s+\omega_{n}\right)^{2}}{\left(s+\alpha \omega_{n}\right)\left(s+\omega_{n} / \alpha\right)}
$$

and choose the compensator $p_{0}(s)$ as

$$
p_{0}(s)=F_{n}^{-1}(s)-1=\frac{[2-(\alpha+1 / \alpha)] \omega_{n} s}{\left(s+\alpha \omega_{n}\right)\left(s+\omega_{n} / \alpha\right)}
$$

Let $k$ be the gain reduction of $F_{n}^{-1}\left(s=j \omega_{n}\right)$, then

$$
\alpha=k^{-1}\left[1+\sqrt{1-k^{2}}\right]
$$

Determination of the filter parameters $\left\{\alpha, \omega_{n}\right\}$ is illustrated in Fig.3; from which it is clear that some fine tunings may be needed to ensure the notch is properly matched. By choosing (3.11), two independent degrees of freedom, namely, frequency $\omega_{n}$ and gain reduction $k$, are imbedded to tune for the most appropriate settings, according to the severity of the actuator saturation.

A digital equivalent in form of (3.10) is

$$
F_{n}^{-1}\left(z^{-1}\right)=\frac{\left(1-p z^{-1}\right)^{2}}{\left(1-\alpha z^{-1}\right)\left(1-\beta z^{-1}\right)}
$$

with $z^{-1}$ as the backward shift operator. Let the sampling interval be $h$, the filter parameters are related to the discrete frequency, $0 \leq \omega_{n} h \leq \pi$, and gain reduction $k$, through the following expressions:

$$
\cos \left(\omega_{n} h\right)=\frac{2 p}{1+p^{2}} \Leftrightarrow p=\frac{1-\cos \left(\omega_{n} h\right)}{\sin \left(\omega_{n} h\right)}
$$

and with $c=\cos \left(\omega_{n} h\right), s=\sin \left(\omega_{n} h\right)$ and $k_{1}=[k(1+s)]^{-1}$, then

$$
k=\frac{2 s}{1+s} \frac{1-\alpha c}{1-2 \alpha c+\alpha^{2}}, \beta=\frac{c-\alpha}{1-\alpha c}
$$

$$
\begin{aligned}
& \alpha=c\left[1-s k_{1}\right]+s \sqrt{k_{1}^{2}-\left[1-s k_{1}\right]^{2}} \\
& \beta=c\left[1-s k_{1}\right]-s \sqrt{k_{1}^{2}-\left[1-s k_{1}\right]^{2}}
\end{aligned}
$$

That is, given $\left\{\omega_{n} h, k\right\}$ for Fig. 3 and $\{\alpha, \beta, p\}$ in (3.13) are uniquely determined and vice versa. The compensator corresponding to $F_{n}^{-1}\left(z^{-1}\right)$ in (3.13) is then given by

$$
p_{0}\left(z^{-1}\right)=\frac{-(p-\alpha)^{2}}{1-\alpha c} \frac{z^{-1}\left[c-z^{-1}\right]}{\left(1-\alpha z^{-1}\right)\left(1-\beta z^{-1}\right)}
$$

The unit delay in $p_{0}\left(z^{-1}\right)$ ensures realizability of (3.15).

\section{Further Designs of Compensators}

The two compensator designs in (3.2) and (3.6), with $p_{0}$ given in (3.11) or (3.15), are simple and immediately applicable. On the other hand, the inherent restrictions, of either forcing $\sigma\left[S_{G E}\right] \equiv 1$ or making $\bar{\sigma}\left[S_{G E}\right] \leq 1$ alone, do not trade off between $S_{G E}$ and the complementary sensitivity function ( $I$ $\left.S_{G E}\right)$, nor take into account the spread between $\bar{\sigma}\left[S_{G E}\right]$ and $\underline{\sigma}\left[S_{G E}\right] \quad\left(=\min \left\{\sigma\left[S_{G E}\right]\right\}\right)$. If the condition number, $\operatorname{cond}\left(S_{G E}\right) \quad\left(=\bar{\sigma}\left[S_{G E}\right] / \underline{\sigma}\left[S_{G E}\right]\right)$ is too large, then the saturation effects will be difficult to alleviate. This is made clear from (2.11),

$$
\left.\begin{array}{rl}
\Delta y= & G[I+K G]^{-1}[I+P] \delta=G S_{G E} \delta \\
\text { Since } \quad & \bar{\sigma}\left[G S_{G E}\right] \leq \bar{\sigma}[G] \bar{\sigma}\left[S_{G E}\right] \\
& \underline{\sigma}\left[G S_{G E}\right] \geq \underline{\sigma}[G] \underline{\sigma}\left[S_{G E}\right]
\end{array}\right\}
$$

i.e., $1 \leq \operatorname{cond}\left(G S_{G E}\right) \leq \operatorname{cond}(G)$ cond $\left(S_{G E}\right)$ (4.3) Therefore the minimum condition number for the saturation dynamics of (4.1) is just that of the open-loop plant, if the compensator is chosen such that $\operatorname{cond}\left(S_{G E}\right) \equiv 1$, i.e., all the singular values of $S_{G E}$ are equal.

This section discusses two expositions for the general question on how to design the compensators for given specifications of the singular values of $S_{G E}(s)$. The developments are more of a theoretic interest than immediate applications.

Assume a desirable $\Sigma_{G E}$ is known and specified for $S_{G E}$. Then in theory, the designs of compensator $P$ may be achieved in either of the two following manners.

Design I (all $\sigma\left[S_{G E}\right]$ may be equal)

Let $I_{s}$ be the skew-identity matrix, i.e., the $90^{\circ}$ rotation of the identity matrix about its centre. $I_{s}$ is unitary and $I_{s}^{2} \equiv I$. Geometrically, it flips a matrix left-right (fliplr) or up-down (flipud).

$$
\begin{aligned}
& \text { In (3.5), if } V_{K G}{ }^{H} V_{P} \equiv I_{s} \text {, i.e., } \\
& V_{P}=V_{K G} I_{s}=\operatorname{flip} \operatorname{lr}\left(V_{K G}\right) \\
& \Sigma_{K G P}=\Sigma_{K G} V_{K G}^{H} V_{P} \Sigma_{P}^{-1}= \\
& {\left[\begin{array}{ccc}
\bar{\sigma}_{K G} & & 0 \\
& \ddots & \\
0 & & \underline{\sigma}_{K G}
\end{array}\right]\left[\begin{array}{lll}
0 & & 1 \\
& \therefore & \\
1 & & 0
\end{array}\right]\left[\begin{array}{ccc}
\sigma_{P}^{-1} & & 0 \\
& \ddots & \\
0 & & \bar{\sigma}_{P}^{-1}
\end{array}\right]=}
\end{aligned}
$$




$$
=\operatorname{diag}\left\{\bar{\sigma}_{K G} \bar{\sigma}_{P}^{-1}, \cdots, \underline{\sigma}_{K G} \underline{\sigma}_{P}^{-1}\right\}
$$

and

$$
S_{G E}=U_{K G} \Sigma_{K G P} U_{P}^{H}
$$

so that $\Sigma_{G E}=\Sigma_{K G P}$ up to some reordering; each $\sigma_{P}=\sigma_{K G} / \sigma_{G E}$ is individually determined and $\Sigma_{P}=\operatorname{diag}\left\{\sigma_{P i}\right\}, i=1 \ldots n$. By choosing $U_{P}=U_{K G}, S_{P}$ is obtained as

$$
S_{P}=U_{K G} \Sigma_{P} \text { flipud }\left(V_{K G}^{H}\right)
$$

and the compensator $P=S_{p}^{-1}-I$.

While theoretically $S_{P}$ of (4.7) exists, its physical realization is usually not feasible because of the irrational dependence of $U_{K G}(s)$ and $V_{K G}(s)$ on $s$. Approximations shall have to be resorted to $[6,4]$. Further developments are needed in the exploration of realizing the compensator thus obtained.

Design II (all $\sigma\left[S_{G E}\right]$ are unequal)

In (3.5), if $V_{K G}{ }^{H} V_{P} \equiv I$, so that

$$
S_{G E}=U_{K G} \Sigma_{K G} \Sigma_{P}^{-1} U_{P}^{H}
$$

and $\Sigma_{G E}=\Sigma_{K G} \Sigma_{P}^{-1}=\operatorname{diag}\left\{\bar{\sigma}_{K G} \underline{\sigma}_{P}^{-1}, \cdots, \underline{\sigma}_{K G} \bar{\sigma}_{P}^{-1}\right\}$

It is emphasized that in (4.5), each $\sigma\left[S_{G E}\right]$ can be made equal so that $\operatorname{cond}\left(S_{G E}\right)=1$. However, in (4.9), $\operatorname{cond}\left(S_{G E}\right)$ is always greater than unity unless $\operatorname{cond}\left(S_{K G}\right)=\operatorname{cond}\left(S_{P}\right)=1$. Thus in general, each $\sigma\left[S_{G E}\right]$ cannot be made equal using (4.9).

Which leads to $\quad S_{P}=U_{K G} \Sigma_{P} V_{K G}^{H}$

where $\Sigma_{P}=\operatorname{diag}\left\{\sigma_{P i}=\sigma_{K G(n-i+1)} / \sigma_{G E(n-i+1)}, i=1 \ldots . n\right\}$, and the compensator $P=S_{p}^{-1}-I=V_{K G} \Sigma_{P}^{-1} U_{K G}{ }^{H}-I$.

As with (4.7), $S_{P}$ of (4.10) has difficulties in its realization. This design gives a Reverse-Frame-Normalizing compensator, very much like the $R F N$ controller in [4]. With slight modifications in the least-square projection procedures of [4], a realizable approximation of $S_{P}$ can be obtained.

\section{Remark}

In (4.1), if $G S_{G E}$ is diagonal, then all the saturation effects are confined to each loop only. Barring this, crosscompensation terms in $P$ will be required. Conversely, $P$ may be chosen so that it diagonalizes $G S_{G E}$, giving $\Delta y_{i}=H_{i}(s) \delta_{i}, i=1 \ldots n$, and each $H_{i}(s)$ is individually specified. Example of $H_{i}(s)$ is $a_{i} /\left(1+s T_{i}\right)$, with gain $a_{i}$ and time constant $T_{i}$ properly tuned to suit saturation levels.

In compensator designs from singular value decompositions, it is implicitly assumed that the system directions, $\left\{U_{K G}, V_{K G}, \ldots\right\}$, remain unchanged. This however is not the case in the presence of saturation and the preservation of control directions is important [5,7]. A usual practice is to insert a directionality block in front of the actuators, see [7] for details (Fig.1). This method is adopted in the following example.

\section{Example}

The following example was used in [7] to show the application of an optimal MIMO discrete compensator, developed through elaborate arguments. Comparisons using the two simple designs in $\S 3$ will be made against the optimal dynamic compensator to show that, even without considering optimality, these SVD designs can produce highly compatible results as well.

The open-loop plant is described by

$$
\begin{aligned}
& A=\left[\begin{array}{cccc}
9.853 e-1 & 9.706 e-3 & 9.737 e-5 & 9.682 e-3 \\
1.956 e-2 & 9.706 e-1 & 1.951 e-2 & .936 e-4 \\
9.705 e-5 & 4.804 e-3 & 9.802 e-1 & 9.657 e-3 \\
9.536 e-3 & -1.436 e-2 & -1.448 e-4 & 9.513 e-1
\end{array}\right] \\
& B=\left[\begin{array}{llll}
9.926 e-3 & 1.969 e-4 & 9.901 e-3 & 4.796 e-5 \\
8.841 e-5 & 9.952 e-5 & 9.989 e-3 & 1.756 e-2
\end{array}\right]^{T} \\
& C=\left[\begin{array}{rrrr}
0 & 2.4 & -3.1 & 1.0 \\
1.0 & 6.0 & -0.5 & -2.8
\end{array}\right], \quad D=\left[\begin{array}{ll}
0 & 0 \\
0 & 0
\end{array}\right]
\end{aligned}
$$

and the linear controller is

$$
\begin{array}{ll}
A_{c}=\left[\begin{array}{cc}
0.9737 & 0.01410 \\
-0.07108 & 1.015
\end{array}\right], \quad C_{c}=\left[\begin{array}{cc}
-1 & 1 \\
2 & -1
\end{array}\right] \\
B_{c}=\left[\begin{array}{cc}
-0.2993 & 0.03026 \\
-0.6821 & 0.1081
\end{array}\right], \quad D_{c}=\left[\begin{array}{ll}
0 & 0 \\
0 & 0
\end{array}\right]
\end{array}
$$

The reference input is $w=\left[\begin{array}{ll}0.3 & 0.3\end{array}\right]^{T}$ and all control bounds are \pm 1 . The uncompensated system is unstable [7].

A plot of $\bar{\sigma}\left[S_{K G}\left(e^{-j \omega h}\right)\right]$ is shown in Fig.4, with an $H_{\infty}$ norm of $14.96 \mathrm{~dB}$ at frequency $\omega_{n} h=0.02666$ [2]. Notch filter (3.13) is applied with $\{\alpha=0.9975, \beta=0.6422, p=0.8602\}$ for $k=-12 \mathrm{~dB}$ at $\omega h=0.025 .\left|F_{n}^{-1}\left(e^{-j \omega h}\right)\right|$ is shown in Fig.4 with $\bar{\sigma}\left[S_{G E}\left(e^{-j \omega h}\right)\right]$, whose $H_{\infty}$-norm is $2.98 \mathrm{~dB}$ at $\omega_{n} h=0.0268$. The resultant compensator from (3.15) is

$$
P\left(z^{-1}\right)=\frac{-1.3651 z^{-1}+1.3805 z^{-2}}{1-0.3553 z^{-1}-0.6406 z^{-2}}\left[\begin{array}{ll}
1 & 0 \\
0 & 1
\end{array}\right]
$$

which is much simpler than the 6-state cross-coupled compensator of $P=K G$.

The system output using (5.3) is shown in Fig.5. Except for a larger overshoot, its response is compatible with the optimal compensator. To further explore the applicability of (3.13), the performance index of accumulated sum of square errors, $\left.J(m)=\Sigma_{t=1 \ldots m}<e(t), e(t)\right\rangle$, is evaluated over the parameter space $\left\{0 \leq \omega_{n} h \leq \pi ; 0<k \leq 1\right\}$ of the notch filter. Surface $J(m=1000)$ is shown in Fig. 6 and as to be expected, its valley approximately matches the hump of $\bar{\sigma}\left[S_{K G}\right]$ in Fig.4. This supports the notion in using SVD treatment for satisfactory saturation compensations.

Fig.7 shows the system outputs using the notch filter for $k=16 \mathrm{~dB}$ at $\omega h=0.15$, and a direct application of compensator (3.2). Both responses seem to challenge that using the optimal compensator. The indices are

\begin{tabular}{|l|c|c|c|c|}
\hline$P$ using & $(5.3)$ & $K G$ & optimal & $k=-16 \mathrm{~dB}$ \\
\hline$J(1000)=$ & 17.75 & 16.31 & 15.77 & 14.63 \\
\hline
\end{tabular}

That is, the notch-filter compensator can be tuned to give even better overall performance results than the optimal compensator developed by [7]. 


\section{Conclusion}

This paper studies the actuator saturation compensation of MIMO systems through singular value decompositions. By establishing a basic expression relating the sensitivity functions of the uncompensated system, the compensator and the compensated system, four compensator designs are proposed. Two simpler ones are of immediate practical success. The other two are more complicated and many possibilities exist for their approximate realizations. A simulated example shows that the two simple designs can produce highly compatible results compared with an elaborate optimal dynamic compensator.

\section{References}

[1] Chen, C.Y. and M.H. Perng, Optimal anti-windup control of saturating discrete-time MIMO systems, Int. J. Control vol.67 no.6 pp. 933-959 Aug 1997

[2] Foias, C., B.Francis, J.W.Helton, H.Kwakernaak, J.B.Pearson, $H_{\infty}$-Control Theory, Springer-Verlay, Berlin 1991

[3] Hui, K, and C.W. Chan, Control Synthesis versus Saturation Compensation for Systems with Rate and Amplitude Constraints, Proc. ACC pp. 1473-1477 June 1998

[4] Hung, Y.S. and A.G.J. MacFarlane, Multivariable Feedback: $A$ Quasi-Classical Approach, Springer-Verlag Berlin 1982

[5] Kothare, M.V., P.J. Campo, M. Morari and C.N. Nett, $A$ Unified framework for the study of anti-windup designs, Automatica vol.30 n.12 pp.1869-1883 1994

[6] Maciejowski, J.M., Multivariable Feedback Design, 1989 Addison-Wesley

[7] Park, J.K. and C.H. Choi, Dynamical Anti-Reset Windup Method for Discrete-Time Saturating Systems, Automatica vol.33 no.6 pp.1055-1072 1997

[8] Tyan, F. and D.S. Bernstein, Anti-Windup Compensator Synthesis for Systems with Saturation Actuators, Int. J. Rob. Non. Cont. vol.5 pp.521-537 1995

[9] Walgama, K.S. and J.Stemby, Conditioing technique for multiinput multioutput processes with input saturation, IEE Proc.-D vol.140 no.4 pp.231-241 1993

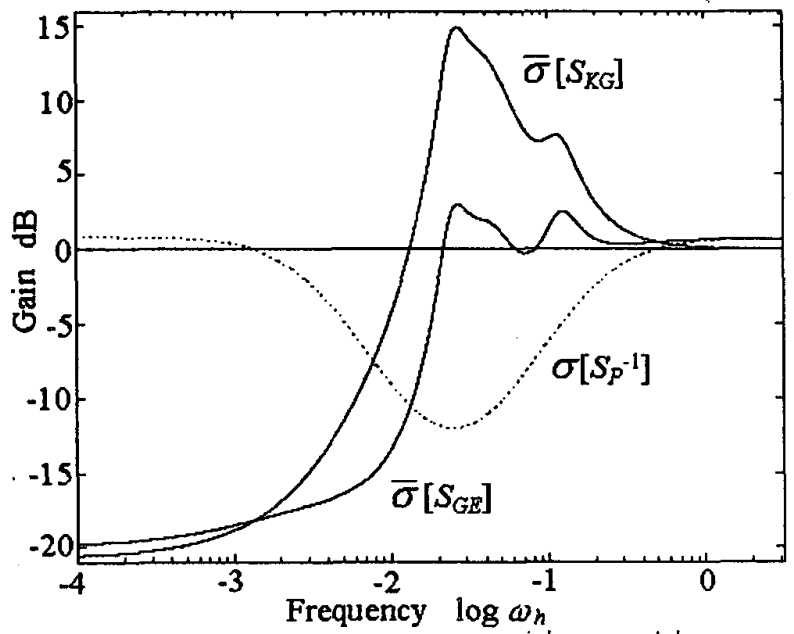

Fig.4 Largest singular value of $S_{K G}\left(e^{j \omega h}\right), S_{G E}\left(e^{j \omega h}\right)$ and $12 \mathrm{~dB}$ notch filter @ $\omega_{h}=0.025$

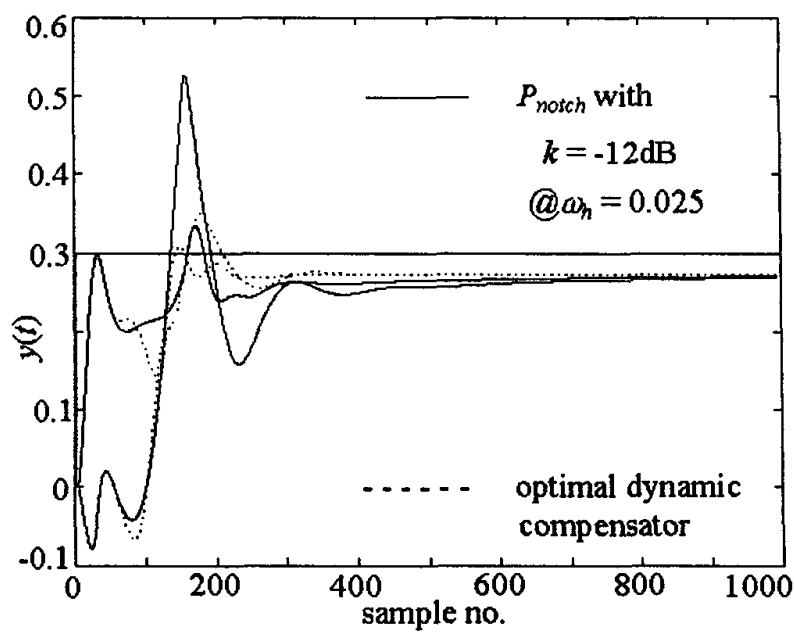

Fig.5 System output using notch filter from Fig.4.

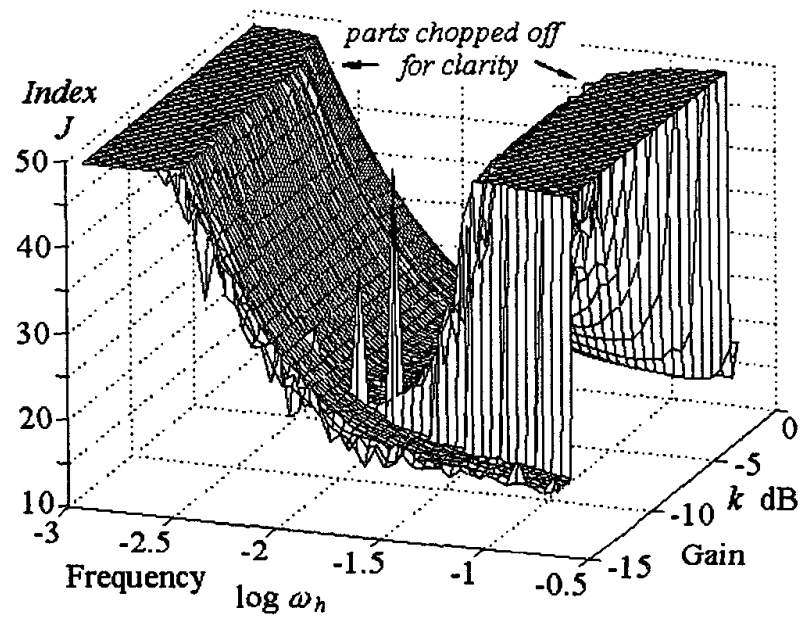

Fig.6 Performance surface as a function of notch filter

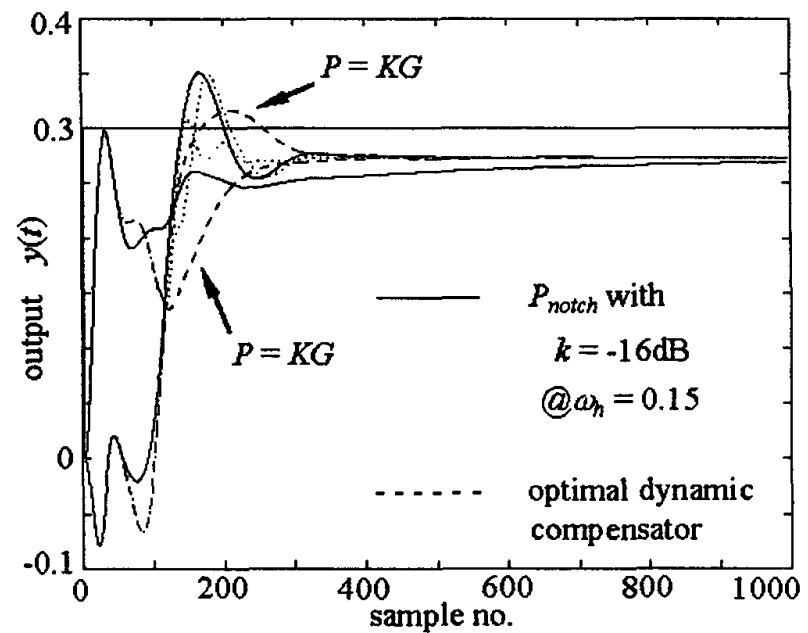

Fig.7 System outputs with $-16 \mathrm{~dB}$ notch filter and $\sigma\left[S_{G E}\left(e^{j \omega h}\right)\right] \equiv 1$ vs. optimal dynamic compensator 